\title{
The Endocannabinoid System: Association with Metabolic Disorders and Tobacco Dependence
}

\author{
C. N. Unachuku MBBS, FWACP \\ Department of Medicine, university of Port Harcourt Teaching Hospital, Port Harcourt
}

\begin{abstract}
Background: Obesity and smoking are major health problems globally as they are risk factors for cardiovascular disease. There appears to be a link between obesity and smoking which is manifested in the endocannabinoid system. This review aims to highlight the physiologic role of the endocannabinoid system and its possible therapeutic applications.

Methods: Literature on the endocannabiniod system was reviewed using medline/pubmed and bibliographic searches for English language studies.

Result: The endocannabiniod system is a physiological system that plays a significant role in the regulation of body metabolism, especially glucose and fat metabolism in addition to nicotine dependence with promising therapeutic application.

Conclusion: The discovery and insights into the functioning of the endocannabiniod system have made a way for new and effective therapeutic approaches. These therapies may provide an effective treatment for obesity and related risk factors for diabetes and cardiovascular disease as well as help people to stop smoking.
\end{abstract}

KEYWORDS: Endocannabiniod system; Obesity; Tobacco dependence.

Paper accepted for publication 20th June 2006.

\section{INTRODUCTION}

The recently discovered endocannabinoid system is a physiological system that plays an important role in the regulation of food intake as well as the storage and metabolism of lipids and glucose. There is a positive association between an over activated endocannabinoid system with obesity and metabolic disorders ${ }^{1,2}$. The endocannabinoid system is also associated with nicotine dependence in smokers ${ }^{1,2}$.

The discovery of the endocannabinoid system dates back to over 5000 years of the history of medicine. Cannabinoids comprise of the various organic substances in cannabis (marijuana). The herbal cannabinoid "delta 9 tetrahydrocannabinol" (THC) is the major active component of marijuana. The membranes of certain nerve cells in the human brain contain receptors for $\mathrm{THC}$ and once bound trigger series of cellular reactions that produce the effects of marijuana 1 .

In 1990, one of these cannabinoid receptors cananbinoid receptor type 1 (CB 1 receptor) was identified in rodents and human. Cannabinoid receptors are present in the membrane of cells of the nervous system as well as fat tissue and muscle cells.

The discovery of receptors to THC suggests that our body produces endocannabinoids that bind to cannabinoid receptors.

In 1992, an endocannabinoid was identified for the first time as a fatty-acid-like substance. The principal endocannabinoids include anandamide and 2arachidonyl glycerol ${ }^{2}$. These substances also bind to and activate the CB 1 receptors.

\section{ROLE OF ENDOCANNABINOIDS \\ Endocannabinoids and Body Weight}

Though the discovery of endocannabinoids was an important step, their functions remained elusive. A clue to their role arose from the known effects of the herbal cannabinoid THC in stimulating appetite. It is suspected that endocannabinoids might also be involved in the regulation of our feeding behaviour ${ }^{3}$ as elevated levels of endocannabinoid are found during hunger and decreased levels noticed after eating. These changes are noticed in the gastrointestinal tract as well as the hypothalamus an area of the brain which serves as a regulator to our feeding behaviour?

Thus endocannabinoids in the brain regulate eating by inducing the feeling of hunger and increasing the motivation to eat.

Researchers using mice as models have discovered that mice lacking the CB 1 receptors were slimmer than their normal controls. In addition, they were resistant to obesity induced by high fat diet ${ }^{4}$. Conversely, mice with CB1 receptors fed with high-fat diets over a long period became obese and exhibited the typical features of obesity i.e. Insensitivity to insulin and high level of blood lipids. Thus, the animals appeared to suffer from the metabolic syndrome seen in human characterized by a cluster of features including abdominal obesity, elevated blood pressure, and insensitivity to insulin and glucose intolerance ${ }^{4}$.

It has been recently discovered that endocannabinoids also regulate the function of 
adipocytes (body energy stores as fat) as well as the balance of body lipids and glucose.

Whereas, endocannabinoids stimulate the mobilization of fat from cells, blocking CB 1 receptors and thereby preventing their action, stimulates the production of adinopectin a beneficial protein capable of improving insulin sensitivity and lipid metabolism. Blocking the CB 1 receptors will therefore reduce over activity of the endocannabinoid system leading to metabolic improvements and weight loss ${ }^{2}$.

\section{Endocannabinoids and Nicotine}

Nicotine, the active ingredient of tobacco stimulates some areas of the brain by increasing levels of dopamine (a neurotransmitter). The area of the brain involved in motivation to seek sweet and palatable food is the same area that is involved in nicotine craving in smokers ${ }^{5}$. Therefore, endocannabinoids are essential for the stimulating effect of tobacco dependence. Hence, dampening the action of CB 1 receptors have been found to alleviate nicotine dependence ${ }^{6}$.

\section{Promising Therapeutic Implications}

The over-activated endocannabinoid system in obese rodents can be dampened by treatment with "cannabinoid receptor antagonists" which block the function of the CB 1 receptor. The cannabinoid receptor antagonist rimonabant, developed by Sanofi-Aventis is the first CB 1 blocker in clinical development ${ }^{2}$.

Promising results have been obtained from the Phase III clinical trials in obesity and associated risk factors for diabetes and cardiovascular disease. Treatment with rimonabant resulted in weight loss, reduced abdominal fat, increased insulin sensitivity and improved lipid and glucose metabolism. It also doubled the chances of successfully stopping smoking, while preventing the weight gain often associated with smoking cessation.

\section{CONCLUSION}

The discovery and insights into the functioning of the endocannabiniod system have made a way for new and effective therapeutic approaches. Preventing the activation of the cannabinoid receptor CB 1 through treatment with receptor blockers like rimonabant may provide an effective treatment for obesity and related risk factors for diabetes and cardiovascular disease as well as help people to stop smoking.

\section{REFERENCES}

1. Piomelli $D$. The molecular logic of endocannabinoid signalling. Nat Rev Neurosci 2003; 4:873-84.

2. Lutz B. The endocannabinoid system: linking body weight, metabolic disorders and tobacco dependence. Diabetes voice 2005; 50: 33 - 35 .

3. Cota D, Marsicano G, Lutz B, Vicennati V, Stalla GK, Pasqualli R, Pagotto U. Endogeneous cannabinoid system as a modulator of food intake. Int J Obes Relat Metab Disord 2003; 27: 289 - 301.

4. Ravinet Trillou C, Armnone M, Delgorge C, Gonalona N, Keane P, Maffrand JP, Soubrie P. Anti-obesity effect of SR141716, a CB 1 receptor antagonist, in diet-induced obese mice. Am J Physiol Regul Integr Camp Physiol 2003; 284:345-53.

5. Targher $\mathrm{G}$. How does smoking affect insulin sensitivity. Diabetes voice 2005; 50: 23 - 25 .

6. Le Foll B, Golderg SR. Cannabinoid CB 1 receptor antagonist as a promising new medication for drug dependence. JPharmacol Exp Ther 2005; 312:875-83.

7. Van Gaal LF, Risanen AM, Scheen AJ, Ziegher O, Rossner S. Effects of the cannabinoid-1 receptor blocker rimonabant on weight reduction and cardiovascular risk factors in overweight patients: 1 year experience from the RI0-Europe study. Lancet 2005; 365: 1389 - 97. 